\section{Tumor-Vakzinierung: klinische Aspekte}

\author{
S. Krenzer ${ }^{1}$, S. Jahn ${ }^{2}$ \\ 1 Study Manager, Merck Serono GmbH, Darmstadt \\ 2 Director Medical Affairs, Merck Serono GmbH, Darmstadt
}

Bei der Entstehung einer Tumorerkrankung sind mehrere Faktoren von Bedeutung. Insbesondere wird dem Immunsystem eine wesentliche Rolle bei der Steuerung einer Krebserkrankung zugeschrieben. Seit Langem ist bekannt, dass das Immunsystem in der Lage ist, entartete Zellen zu erkennen und diese auch erfolgreich zu bekämpfen. Allerdings bleiben Tumorzellen oft vom Immunsystem „unentdeckt“, da diese sich aus körpereigenen Zellen entwickeln und der Tumor selbst fortlaufend neue Mechanismen entwickelt, um der Kontrolle durch das Immunsystem zu entkommen. Klassischerweise erfolgt die Behandlung einer Tumorerkrankung durch die operative Entfernung des Tumors, die Anwendung von Chemotherapie und/oder von Strahlentherapie. Allerdings sind die Nebenwirkungen vor allem bei der Chemound der Strahlentherapie sehr hoch. Deshalb wird seit Jahren nach neuen Therapiemöglichkeiten gesucht. Einen vielversprechenden Ansatz stellt hierbei die Immuntherapie dar, die Krebszellen mithilfe des Immunsystems zerstört und zusätzlich noch durch ihre Spezifität vor allem eine Verbesserung des Nebenwirkungsprofils hervorruft.

Seit geraumer Zeit steht die Frage im Raum, ob die TumorVakzinierung tatsächlich den „Stein der Weisen“ darstellt und ob sich mit dieser Therapieoption die therapeutischen Möglichkeiten zur Behandlung einer Krebserkrankung immens verbessern können. Warum aber wird der Tumor-Vakzinierung eine so hohe Erfolgsquote zugeschrieben?

Bei der Vakzinierung ist zunächst die präventive von der therapeutischen zu unterscheiden. Die präventive Vakzinierung - so wie sie ihren Einsatz in der Klinik findet - ist jedem aus der Impfung gegen die stets bekannten Krankheiten wie Röteln, Masern oder Mumps bekannt. Seit 2006 stehen aber auch Impfstoffe zur Prävention von Gebärmutterhalskrebs zur Verfügung. Diese Impfstoffe sind gegen bestimmte Typen humaner Papillomviren gerichtet, die zu einem hohen Prozentsatz für die Entstehung von Gebärmutterhalskrebs verantwortlich sind. Durch die Impfung kann maßgeblich dazu beigetragen werden, die Zahl der Erkrankungen an Gebärmutterhalskrebs zu reduzieren.

Liegt hingegen schon eine Krebserkrankung vor, so kommt die therapeutische Vakzinierung zum Einsatz. Hierbei ist die passive von der aktiven Immunisierung zu unterscheiden. Unter der passiven Immunisierung ist vor allem die Anwendung monoklonaler Antikörper zu verstehen sowie die Verwendung von gegen Krebs gerichteten Immun-(T-)Zellen (sog. TILs). Eine Behandlung mit monoklonalen Antikörpern ist im Grunde nicht direkt als Immuntherapie zu verstehen, da es sehr schwierig ist, spezifische Merkmale auf Tumorzellen zu identifizieren, die nicht auf gesundem Gewebe vorkommen. Die Antikörpertherapie besteht vielmehr darin, in bestimmte Signalwege von Tumorzellen einzugreifen, sodass das vermehrte Wachstum des Tumors verhindert werden kann (z. B. durch Cetuximab, Trastuzumab), bestimmte Immunreaktionen ausgelöst werden können (z. B. durch Rituximab) oder die Versorgung des Tumors gehemmt werden kann durch Hemmung der Blutgefäßneubildung (z.B. durch Bevacizumab). Heutzutage kommt die zielgerichtete Antikörpertherapie gegen ein bestimmtes Tumorantigen meist in Kombination mit der unspezifischen zytotoxischen Chemotherapie zum Einsatz. Da dieser Therapieansatz auch eine Immunantwort generieren kann, wird momentan gerade der Ansatz untersucht, eine zielgerichtete Antikörpertherapie mit einer Immuntherapie zu verknüpfen. Hierzu sind bereits erste klinische Studien durchgeführt worden.

Bei der aktiven Immunisierung werden zurzeit mehrere Ansätze verfolgt. Eine Vakzinierung kann mit Tumorzellen oder Antigenen oder Peptiden von einer Tumorzelle stattfinden, sie kann aber auch mit genetisch modifizierten Tumorzellen selber oder mit der Unterstützung durch dendritische Zellen (DC) erfolgen. Das erste therapeutische Krebsvakzin, das von der FDA zugelassen wurde, ist Sipuleucel-T, das zur Behandlung des Prostatakarzinoms verwendet wird. Hierbei handelt es sich um eine personalisierte Therapie, da dem Patienten DCs entnommen werden, die ex vivo mit dem Fusionsprotein PA2024 inkubiert werden und nach einer Waschung dem Patienten wieder infundiert werden. Das Fusionsprotein PAP2024 besteht aus der prostataspezifischen sauren Phosphatase (PAP) und dem Granulozyten-Makrophagen-koloniestimulierenden Faktor (GM-CSF). Die dadurch induzierten T-Zellen sind nun gegen das Tumorantigen PAP gerichtet und bekämpfen somit die Tumorzellen, die dieses Tumorantigen (PAP) exprimieren. PAP wird hauptsächlich von Tumorzellen exprimiert, nicht aber oder nur zu einem geringen Anteil von gesunden Zellen.

Gegenüber der konventionellen Tumortherapie kann die Immuntherapie langfristig das Tumorwachstum besser kontrollieren; zusätzlich weist sie noch eine bessere Verträglichkeit für den Patienten im Vergleich zu den bekannten zytotoxischen Therapien auf. Dennoch aber erweist es sich teilweise als nicht trivial, das richtige Design sowie die richtige Dosierung in einer Studie mit einem Immuntherapeutikum zu finden. Somit sind hier noch viele Hürden zu überwinden, um erfolgreich weiter den Weg in der Entwicklung immuntherapeutischer Ansätze zu gehen.

Nach wie vor bleibt es aber unumstritten, dass der medizinische Bedarf nach einer erfolgreichen Tumortherapie uneingeschränkt vorhanden ist. Somit wird stets weiter daran geforscht, die chirurgischen Möglichkeiten zur Tumorentfernung zu verbessern, Chemotherapeutika verträglicher zu machen, Strahlentherapien zu optimieren und neue Ansätze wie beispielsweise die Immuntherapie weiter zu verfolgen.

Interessenkonflikt: Die Autoren sind Mitarbeiter der Merck Serono GmbH.

\section{Bibliografie}

DOI http://dx.doi.org/10.1055/s-0032-1324902 Arzneimittelforschung 2012; 62, Suppl. 1: S8-S8 (c) Georg Thieme Verlag KG Stuttgart · New York ISSN 0004-4172

Please note that the print version was published with a different, incorrect DOI. The correct DOI is the one shown here. 